Pol. Con. (Edición núm. 2) Vol. 1, No 2

Diciembre 2016, pp. 3-13

ISBN: 2550 - 682X

DOI: 10.23857/pc.v2i2.15

Recepción: 30/ 08/ 2016

Aceptación: 13 / 12/ 2016

Publicación: 27/ 02/2017

Ciencias de la educación

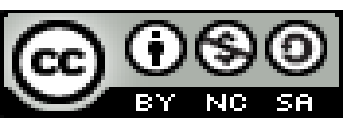

Artículo de investigación

\title{
Una aproximación de los factores de riesgo psicológico y social en el adolescente
}

An approximation of the psychological and social risk factors in the adolescent Uma aproximação de fatores psicológicos e risco social em adolescentes

Uma aproximação dos fatores de risco psicológico e social em adolescente

\author{
Jeanneth E. Balseca-Basantes ${ }^{\mathrm{I}}$ \\ jeannethbalseca@gmail.com
}

Correspondencia: jeannethbalseca@gmail.com

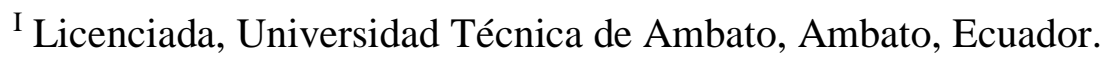




\title{
Resumen
}

La salud integral de los adolescentes depende en particular de su propio comportamiento, que, a su vez, sufre la poderosa influencia del ambiente en que viven. Podemos mencionar entre ellos el consumo de tabaco, alcohol, y otras drogas, con variadas consecuencias a largo plazo: mayores riesgos de desarrollar cáncer y enfermedades cardiovasculares, respiratorias y hepáticas. A estas consecuencias cabe añadir los problemas inmediatos que surgen en relación al consumo de dichas sustancias: aumento del riesgo de lesión accidental, conductas sexuales de riesgo, etc. Urge, por lo tanto, prestar una mayor atención por parte de la comunidad sanitaria a los adolescentes.

Palabras clave: adolescentes; conducta del adolescente; factor de riesgo; psicología del adolescente.

\begin{abstract}
The integral health of adolescents depends in particular on their own behavior, which, in turn, suffers the powerful influence of the environment in which they live. We can mention among them the consumption of tobacco, alcohol, and other drugs, with various consequences to Long term: greater risks of developing cancer and cardiovascular, respiratory and liver diseases. These consequences include the immediate problems that arise in relation to the consumption of these substances: increased risk of accidental injury, risky sexual behavior, etc. It is therefore urgent that the health community give more attention to adolescent.
\end{abstract}

Key words: adolescents; adolescent behavior; risk factor; adolescent psychology.

\section{Resumo}

A saúde geral de adolescentes depende em particular do seu próprio comportamento, o que, por sua vez, sofre a influência poderosa do ambiente em que vivem. Podemos citar entre eles o consumo de tabaco, álcool e outras drogas, com consequências variadas longo prazo aumento do risco de desenvolver câncer e doenças cardiovasculares, respiratórias e doenças do fígado. Estas consequências devem acrescentar os problemas imediatos que se colocam em relação ao consumo destas substâncias: o aumento do risco de lesões acidentais, comportamento sexual de risco, etc. Insta, portanto, uma maior atenção por parte da comunidade de saúde para 
adolescentes.

Palavras chave: adolescentes; comportamento do adolescente; fatores de risco; a psicologia do adolescente.

\section{Introducción}

Las palabras, organizadas en los diferentes sistemas de comunicación verbal que conocemos, son instrumentos de la cultura que permiten la comunicación entre las personas y la existencia misma de la humanidad, pues son portadoras de gran parte del saber acumulado por los seres humanos a lo largo de siglos. Cada palabra puede tener diferentes acepciones y esto enriquece el intercambio entre quienes participen en la comunicación, siempre que esos significados sean compartidos. ${ }^{1}$

Partiendo de la importancia del empleo del término «riesgo» fundamentalmente en la salud pública y ambiental, además en otros sectores importantes de nuestra vida social, con este trabajo se pretende hacer una revisión de los principales factores de riesgo psicosociales que pueden incidir en los adolescentes, el conocimiento de éstos puede servir de ayuda para prevenir la aparición de futuros problemas de salud.

\section{Definición de riesgo}

La OMS define al factor de riesgo como cualquier rasgo, característica o exposición de un individuo que aumente su probabilidad de sufrir una enfermedad o lesión. Entre los factores de riesgo más importantes cabe citar la insuficiencia ponderal, las prácticas sexuales de riesgo, la hipertensión, el consumo de tabaco y alcohol, el agua insalubre, las deficiencias del saneamiento y la falta de higiene. ${ }^{2}$

En las ciencias de la salud el riesgo es entendido como la probabilidad de sufrir una determinada enfermedad o padecimiento que incide directamente en la disminución de la calidad de vida de las personas. Se plantea que en esta y otras áreas del conocimiento el uso de la terminología referida al riesgo es incorrecto e inexacto, y que cuando se habla de factores de riesgo deben cuantificarse, con vista a tener una idea más realista de su significado. Este cálculo debería incluir un ámbito y período determinados, pues sin las referencias espacial y temporal el uso del riesgo no tiene sentido. ${ }^{3}$ 
Actualmente la preocupación de la sociedad por el riesgo está muy relacionada con la complejidad que se vive. La aceleración de los cambios sociales, económicos y políticos, la globalización y la progresiva industrialización traen aparejadas la contaminación ambiental, la escasez de recursos naturales vitales como el agua, accidentes industriales que han socavado la seguridad pública, la proliferación de determinadas enfermedades (tanto en humanos como en animales y plantas), transformaciones irreversibles del medioambiente, entre otras.

El ritmo actual de cambios reduce la estabilidad social e institucional a largo plazo, afecta la facultad de predecir el futuro y, por tanto, aumenta la incertidumbre. Ha aumentado también la conciencia sobre el riesgo, y en consecuencia, la intolerancia hacia este. Pero también las personas se esfuerzan por protegerse de las catástrofes y del efecto de esos riesgos ilocalizables, indefinidos y con dimensiones no previstas. ${ }^{1-4}$

La Psicología, como parte de las ciencias sociales, también ha hecho su aporte al estudio de los factores de riesgo, investigando los comportamientos y los estilos de vida no saludables como factores que colocan a los individuos en situaciones de riesgo. En este caso, más que medir, se trataría de caracterizar estos factores para, conociéndolos, prevenirlos. ${ }^{5}$

La adolescencia, por ser un período crítico sometido a constantes cambios en el desarrollo físico, psíquico y social del adolescente, así como también en el marco familiar en que éste se desenvuelve, se considera como un período de riesgo en el cual pueden darse las bases para la aparición de síntomas y enfermedades, así como también de alteraciones de la personalidad. ${ }^{6}$

La Organización Mundial de la Salud (OMS), define la adolescencia como la etapa que transcurre entre los 11 y 19 años de edad. En cada una de las etapas se presentan cambios fisiológicos, estructurales anatómicos y modificación en el perfil psicológico y de la personalidad. Cada individuo posee una personalidad y en esta etapa es cuando más la manifiesta no solo de manera individual sino de forma grupal, para poder medir el grado de aceptación en los diferentes escenarios sociales e ir haciendo los ajustes o modificaciones necesarios para sentirse aceptado socialmente. $^{7}$

El perfil psicológico generalmente es transitorio y cambiante es emocionalmente inestable. El desarrollo de la personalidad dependerá en gran medida de los aspectos hereditarios, de la 
estructura y experiencias en la etapa infantil preescolar y escolar y de las condiciones sociales, familiares y ambientales en el que se desenvuelva el adolescente. Por la misma inestabilidad emocional, por desconocimiento, temor, experimentan nuevas vivencias, falta de una toma de decisión adecuada y en ocasiones combinados con una baja autoestima, es una etapa para una gran parte de ellos, muy susceptible para tomar una conducta inadecuada. ${ }^{7}$

Las áreas médica y psicológica han tenido desde sus inicios un vínculo estructural muy fuerte tanto en su conocimiento teórico como en la práctica, mediante la creación de herramientas y estrategias que colaboran con las tareas curativas y preventivas de la enfermedad y con el mantenimiento de la salud. La concepción de enfermedad médica y de enfermedad psicológica comprende elementos comunes que se complementan y permiten una comprensión más amplia del complejo fenómeno del proceso salud-enfermedad, al tiempo que brinda herramientas para el manejo integral, bio-psicosocial, de las personas aquejadas por una u otra enfermedad. ${ }^{8}$

\section{Factores psicosociales de riesgo en la adolescencia}

La familia cumple importantes funciones en el desarrollo de sus miembros junto a los demás componentes de la sociedad. Modela sentimientos y ofrece patrones de conductas, dándose o no en su seno las condiciones para alcanzar un adecuado crecimiento y desarrollo. Cuando la familia es disfuncional, no cumple sus funciones básicas y no quedan claras las reglas y roles familiares, se dificulta el libre y sano desarrollo de la personalidad del adolescente. ${ }^{9}$

El grupo es de gran relevancia en la adolescencia, ya que el adolescente siente la necesidad de autoafirmación y del seguimiento del código grupal. Las normas del grupo influyen en los comportamientos individuales, lo que funciona como factor de riesgo o protector, por la potencialidad de estimular conductas positivas o negativas, en correspondencia con sus cánones; estando el adolescente en riesgo de pertenecer a grupos antisociales. ${ }^{9}$

En esta etapa puede surgir diferentes problemáticas como:

- $\quad$ El consumo de sustancias tóxicas, como el alcohol y las drogas, originado en el afán de parecer adultos, los puede llevar a asumir conductas que consideran de mayores pero que son 
riesgosas por su potencialidad de constituirse en adicciones, causales de dependencias y daños a la salud y la sociedad.

- Los conflictos amorosos, problemas docentes, relaciones no adecuadas con los padres, las deformaciones físicas, el rechazo del grupo pueden provocar autovaloración y autoestima negativas originando depresión y/o ansiedad y traducirse en conductas suicidas o lesiones autoinflingidas.

- Una práctica sexual irresponsable por una inadecuada educación sexual conlleva a mayor riesgo de infecciones de trasmisión sexual (ITS) incluyendo el VIH/ SIDA, al aborto, la promiscuidad y la maternidad/paternidad tempranas. El embarazo en estas edades conlleva a una serie de situaciones que pueden atentar contra la salud de la madre y la de su futuro hijo, así como a la frustración de sueños, planes y estudios venideros, y la adolescente y su hijo pasan a depender completamente de su familia.

- $\quad$ El abandono escolar y laboral provoca que el adolescente se encuentre desvinculado de la sociedad y no tenga la posibilidad de una autoafirmación positiva, al disminuir las posibilidades de comprobar sus destrezas para enfrentar los problemas y asumir responsabilidades, lo cual resquebraja su autoestima, la confianza en sí mismo y en sus posibilidades de desarrollo social. Favorece además a la pertenencia a grupos con patrones inadecuados para la sociedad.

Después de lo anterior expuesto se resume que los principales actores de riesgo psicológicos son los siguientes:

- Insatisfacción de las necesidades psicológicas básicas. Dentro de ellas podemos destacar la necesidad de autoafirmación, de independencia, de relación íntima personal y la aceptación por parte del grupo.

- Patrones inadecuados de educación y crianza. Estos pueden ser:

- Sobreprotección: Se puede manifestar de una manera ansiosa (al crear sentimientos de culpa en el adolescente) o de una manera autoritaria (al provocar rebeldía y desobediencia). 
- $\quad$ Autoritarismo: Limita la necesidad de independencia del adolescente y mutila el libre desarrollo de su personalidad, para provocar como respuesta en la mayoría de los casos, rebeldía y enfrentamientos con la figura autoritaria y pérdida de la comunicación con los padres.

- Agresión: Tanto física como verbal, menoscaba la integridad del adolescente, su autoimagen y dificulta en gran medida la comunicación familiar.

- Permisividad: Esta tendencia educativa propicia la adopción de conductas inadecuadas en los adolescentes por carencia de límites claros.

- $\quad$ Autoridad dividida: Este tipo de educación no permite claridad en las normas y reglas de comportamiento, y provoca la desmoralización de las figuras familiares responsables de su educación.

- Ambiente frustrante. Cuando el adolescente no encuentra adecuadas manifestaciones de afecto, cuando hay censura inmotivada y frecuente hacia su persona, cuando se reciben constantes amenazas, castigos e intromisiones en su vida privada y cuando se aprecia un desentendimiento y alejamiento de las problemáticas que presenta. $^{5}$

- $\quad$ Sexualidad mal orientada. Cuando por la presencia de prejuicios en relación con los temas sexuales, la comunicación en esta esfera queda restringida y el adolescente busca por otros medios, no siempre los idóneos, sus propias respuestas e informaciones o en muchos casos mantiene grandes lagunas que le acarrean grandes problemas por el desconocimiento, la desinformación y la formación de juicios erróneos en relación con la sexualidad.

En este mismo orden y dirección, en relación con el riesgo social del adolescente expondremos algunos factores sociales y ambientales que pueden conducir a resultados negativos en los jóvenes. Como principales factores de riesgo social tenemos: ${ }^{6}$

- Inadecuado ambiente familiar. Cuando la familia es disfuncional, no cumple sus funciones básicas y no quedan claros las reglas y roles familiares, se dificulta el libre y sano desarrollo de la personalidad del adolescente. Es necesario que exista un soporte familiar abierto, capaz de asimilar los cambios requeridos para la individualización del adolescente. 
- Pertenencia a grupos antisociales. Este factor tiene como causa fundamental la satisfacción de la necesidad de autoafirmación y la necesidad del seguimiento del código grupal. Por lo general cuando los adolescentes no encuentran una vía adecuada de autoafirmación tratan de buscarla en este tipo de grupo donde fácilmente la encuentran, con el reconocimiento grupal ante la imitación de sus patrones inadecuados.

- La promiscuidad. Es un factor de riesgo social que no sólo puede ser motivo de embarazos precoces y enfermedades de transmisión sexual, sino que también propicia una autovaloración y autoestima negativas que puede deformar la personalidad del adolescente.

- Abandono escolar y laboral. Este hecho provoca que el adolescente se halle desvinculado de la sociedad, y no encuentre la posibilidad de una autoafirmación positiva, al disminuir las posibilidades de comprobar sus destrezas para enfrentar los problemas y asumir responsabilidades, lo cual resquebraja su autoestima, la confianza en sí mismo y en sus posibilidades de desarrollo social.

- Bajo nivel escolar, cultural y económico. Estos son elementos considerados como protectores del desarrollo y la salud y el hecho de presentar un déficit en ellos le impide al adolescente un enfrentamiento adecuado a las situaciones de conflicto.

Estudio realizado por Sanabaria 10 sobre Factores psicosociales de riesgo asociados a conductas problemáticas en jóvenes infractores y no infractores, los resultados muestran que los adolescentes que se encuentran recluidos en dos instituciones para menores infractores presentan una mayor frecuencia de exposición a los factores de riesgo, asociados con la conducta antisocial y delictiva, en comparación con los adolescentes no infractores que asisten a una institución pública, en los niveles exosistema, microsistema y macrosistema. Los dos grupos de adolescentes se encuentran expuestos al maltrato, el consumo y abuso de alcohol en proporciones similares, y constituyen los factores de riesgo que más se asocian a la generación del comportamiento antisocial y el comportamiento delictivo.

En este orden de ideas se puede citar también la investigación de Berrío Acosta G. ${ }^{8}$ con respecto a "Factores de riesgo y aspectos psicosociales de los adolescentes con enfermedad cardiovascular". La revisión documental hizo evidente que en los adolescentes predominan los 
factores de riesgo de tipo social relacionados con el predominio de hábitos alimenticios poco saludables y la escasa actividad física. Quienes padecen enfermedad cardiovascular se preocupan en exceso por su condición de salud. Además, se les dificulta comunicar a los médicos y enfermeras su preocupación o sentimiento de incertidumbre respecto a su condición. Por último, tienen miedo a morir y no son capaces de dialogar con los parientes sobre su problema de salud.

El embarazo a cualquier edad constituye un hecho biopsicosocial muy importante, pero en la adolescencia conlleva a una serie de situaciones que pueden atentar contra la salud de la madre y la de su futuro hijo. Varios autores que describen en sus investigaciones, que en los últimos años los embarazos entre las jóvenes adolescentes de 15 -19 años de edad han aumentado. ${ }^{9}$

En varios estudios se ha observado que los adolescentes ignoran los riesgos y daños que pueden significar las relaciones sexuales desordenadas que además del embarazo puede provocar ITS, la promiscuidad y la prostitución. ${ }^{9}$

En términos generales, en Ecuador, de cada 100 partos, 23 son de adolescentes, el $15.21 \%$ del total de las adolescentes ecuatorianas estuvieron embarazadas o tuvieron al menos un hijo. En el país hay 3.684 niñas entre 12 a 14 años, el 17.2\% de las adolescentes de 15 a 19 años, que ya son madres. (Fuente: Censo de Población y Vivienda 2010). La principal causa de egreso hospitalario fue el embarazo parto y puerperio, que equivale al $79 \%$ de los egresos hospitalarios en mujeres. (Fuente: Egresos Hospitalarios 2010, Estadísticas Vitales MCD). ${ }^{7}$

Resulta oportuno destacar que en la actualidad, existe la mayor ocurrencia de enfermedades de origen biopsicosocial, en especial los trastornos alimentarios, que involucran diferentes sistemas del cuerpo y los aspectos inherentes a la persona y sus relaciones sociales. La mayor incidencia de la anorexia y bulimia nerviosa se presenta entre las niñas en la fase media y final de la adolescencia. Entre los factores que aumentan el riesgo para la aparición de los trastornos alimentarios en la adolescencia se encuentran: la genética, los cambios corporales en la pubertad, la vulnerabilidad de los adolescentes a los ideales de delgadez, la presión social por ser delgada, la insatisfacción con la imagen corporal, la dieta restrictiva, la depresión y la baja autoestima. ${ }^{11}$ 


\section{Consideraciones Finales}

La adolescencia es una de las fases de la vida más fascinantes y quizás más complejas, una época en que la gente joven asume nuevas responsabilidades y experimenta una nueva sensación de independencia. Los jóvenes buscan su identidad, aprenden a poner en práctica valores aprendidos en su primera infancia y a desarrollar habilidades que les permitirán convertirse en adultos atentos y responsables.

El conocimiento y manejo de los factores de riesgo psicológicos y sociales en la salud de los adolescentes permitirá identificar a aquellos adolescentes que están más expuestos a sufrir los daños que los aquejan, como accidentes, embarazos precoces, abuso de sustancias psico-activas, enfermedades de transmisión sexual y el suicidio; por lo que la sociedad y el sistema de salud deben propiciar el desarrollo de factores protectores que apoyen el crecimiento y la maduración sana del adolescente.

\section{Referencias bibliográficas}

1- Echemendía Tocabens Belkis. Definiciones acerca del riesgo y sus implicaciones. Rev Cubana Hig Epidemiol 2011 [citado 25 Feb 2017]; 49(3): 470-481. Disponible en: http://scielo.sld.cu/scielo.php?script=sci_arttext\&pid=S1561-30032011000300014\&lng=es.

2- OMS. Factor de riesgo. 2017 [citado 25 Feb 2017]; Disponible en: http://www.who.int/topics/risk_factors/es/

3- Álvarez R. El método científico en las ciencias de la salud. Las bases de la investigación biomédica. 1ra. ed. Madrid: Díaz de Santos, S.A.; 1996.

4 Mesa Raya C. El concepto de riesgo y la protección social a la infancia en Aragón. Un análisis socio-jurídico. Revista Aragonesa de Administración Pública 2008 [citado 25 Feb 2017]; (33): 247-80.

Disponible

en: http://plan.aragob.es/FBA.nsf/0/69ab51f700828ec3c1257585003bc3de/\$FILE /El\%20concepto\%20de\%20riesgo\%20y\%20la\%20protecci\%C3\%B3n\%20social\%20a\%20la\%20 infancia\%20en\%20Arag\%C3\%B3n\%20un\%20an\%C3\%A1lisis\%20socio -jur\%C3\%ADdico.pdf 
5- Martínez M, Alba LC, Sanabria G. Interrelación dialéctica entre calidad de vida y motivaciones relativas a la salud. Rev Cubana Med Gen Integr. 2010 [citado 25 Feb 2017]; 26(1). Disponible en: http://www.bvs.sld.cu/revistas/mgi/vol_26_1_10/mgi16110.htm

6- Herrera Santi Patricia. Principales factores de riesgo psicológico y social en el adolescente. Rev Cubana Pediatr. 19992010 [citado 25 Feb 2017]; 71(1): 39-42. Disponible en: http://scielo.sld.cu/scielo.php?script=sci_arttext\&pid=S0034-75311999000100006\&lng=es.

7- Del Rocío Calvopiña Parra. Factores de riesgo psicosociales que influyeron en el embarazo y maternidad adolescentes. 2012 [citado 25 Feb 2017]. Disponible en: http://www.dspace.uce.edu.ec/bitstream/25000/2052/1/T-UCE-0007-70.pdf

8- Berrío Acosta G M, Letrado Forero L. Factores de riesgo y aspectos psicosociales de los adolescentes con enfermedad cardiovascular. 2011[citado 25 Feb 2017]. Disponible en: http://www.dspace.uce.edu.ec/bitstream/25000/2052/1/T-UCE-0007-70.pdf

9- García Mendiola J. J. Factores de riesgos biológicos y sociales en los adolescentes del municipio Regla. 2012 [citado 25 Feb 2017]. Disponible en: http://www.codajic.org/sites/www.codajic.org/files/37\%20-

$\% 20$ Factores\%20de\%20riesgos\%20biol\%C3\%B3gicos\%20y\%20sociales\%20en\%20los\%20adol es.pdf

10- Sanabria A M. Factores psicosociales de riesgo asociados a conductas problemáticas en jóvenes infractores y no infractores. 2010 [citado 25 Feb 2017]. Revista diversitas - perspectivas en psicología 6 (2). Disponible en: http://www.redalyc.org/articulo.oa?id=67915140005

11- Portela de Santana M. L., da Costa Ribeiro Junior H., Mora Giral M., Raich R. M. ${ }^{\text {a }}$ La epidemiología y los factores de riesgo de los trastornos alimentarios en la adolescencia: una revisión. Nutr. Hosp. 2012 [citado 25 Feb 2017]; 27(2): 391-401. Disponible en: http://scielo.isciii.es/scielo.php?script=sci_arttext\&pid=S0212-16112012000200008\&lng=es 